\title{
Meson effect in the proto neutron star PSR $\mathrm{J} 0348+0432$
}

\author{
Xian-Feng Zhao ${ }^{1,2}$
}

Received: October 24, 2018/ Accepted: date

\begin{abstract}
The effect of mesons $f_{0}(975)$ (named as $f$ ), $\phi(1020)$ (named as $\phi$ ) and $\delta$ on the moment of inertia of the PNS PSR J0348+0432 is examined in the framework of the relativistic mean field theory considering the baryon octet. It is found that the energy density $\varepsilon$ and pressure $p$ will increase considering the mesons $\delta$ whereas will decrease as the mesons $f$ and $\phi$ being considered. When the mesons $f, \phi$ and $\delta$ are considered, the energy density and pressure will all decrease. It is also found that the contribution of mesons $f, \phi$ and $\delta$ to the central energy density is only the central energy density's $0.06 \sim 0.6 \%$ whereas the contribution of mesons $f, \phi$ and $\delta$ to the central pressure is the central pressure's $4 \sim 7 \%$. For the radius, it will decrease when the contributions of mesons $f, \phi$ and $\delta$ are considered. The moment of inertia $I$ will increase considering the mesons $\delta$ whereas will decrease as the mesons $f$ and $\phi$ being considered. When the mesons $f, \phi$ and $\delta$ are all considered, the moment of inertia will decrease. It is found that the contribution of mesons $f$ and $\phi$ to moment of inertia is $4 \sim 9$ times larger than that of mesons $\delta$. Our results show that the mesons $f, \phi$ and $\delta$ contribute to the moment of inertia's $2 \sim 5 \%$.
\end{abstract}

Keywords moment of inertia $\cdot$ relativistic mean field thoery $\cdot$ neutron star

PACS 26.60.Kp $\cdot 21.65 . \mathrm{Mn}$

\section{Introduction}

The moment of inertia is an important physical quantity for the rotation of a neutron star (NS). Starting from the theory of general relativity, the formula for calculating the moment of inertia of a slowly rotating NS with spherical symmetry was derived by Hartle et al in 1967 and 1968 11,2]. This formula reflects the complex relation between moment of inertia and mass and radius.

\footnotetext{
Xian-Feng Zhao

1 School of Sciences, Southwest Petroleum University, Chengdu, 610500 China

2 School of Electronic and Electrical Engineering, Chuzhou University, Chuzhou 239000, China

E-mail: zhaopioneer.student@sina.com
} 
The moment of inertia of the NS PSR J0737-3039A with the mass of $M=1.338$ $\mathrm{M}_{\odot}$ was studied with five different kinds of equations of state by Bejger et al in 2005 [3]. The moment of inertia they determined is in the range $0.98 \times 10^{45} \sim$ $1.72 \times 10^{45} \mathrm{~g} \mathrm{~cm}^{2}$. It was further identified as $I=1.3 \times 10^{45} \mathrm{~g} \mathrm{~cm}^{2}$ by Raithel et al in 2016 四. In their work, they calculated the radii and moments of inertia of the NS PSR J0737-3039A by 41 different equations of state.

Last several years, two massive NSs have been successively discovered. In 2010, the massive NS PSR J1614-2230 with mass of $1.97_{-0.04}^{+0.04} \mathrm{M}_{\odot}$ was found by Demorest et al [5]. Two years later, another more larger mass NS PSR J0348+0432 with the mass of $\mathrm{M}=2.01 \pm 0.04 \mathrm{M}_{\odot}$ was discovered by Antoniadis et al $\sqrt{6}$. Thereafter, many theoretical studies have been carried out on them $[$, $8,9,10,11,12,13,14,15$, 16.

For the calculations of the NS matter, the relativistic mean field (RMF) theory is a better method [17]. In its early application, only mesons $\sigma, \omega$ and $\rho$, by which only the interactions between nucleons can be described, were considered [1] . Later, mesons $f_{0}(975)$ (named as $f$ ) and $\phi(1020)$ (named as $\phi$ ) were introduced to describe the interaction between hyperons [19. In order to describe the isospin asymmetry properties of NS matter, mesons $\delta$ also needs to be introduced [20]. We call this the $\sigma \omega \rho f \phi \delta$ model.

The proto neutron star (PNS), which emits energy by neutrino radiation, is a very important celestial body for the understanding of the stellar evolution [21]. Will the introduction of the new mesons have a great impact on the properties of the PNS? We are interested in this issue.

In this paper, the effect of mesons $f_{\mathrm{s}}, \phi \mathrm{s}$ and $\delta \mathrm{s}$ on the moment of inertia of the PNS PSR J0348+0432 is examined in the framework of the RMF theory considering the baryon octet.

\section{The RMF theory of a PNS}

The Lagrangian density of hadron matter containing mesons $f, \phi$ and $\delta$ reads as follows $19,22,23,24$

$$
\begin{aligned}
\mathcal{L}= & \sum_{B} \bar{\Psi}_{B}\left(i \gamma_{\mu} \partial^{\mu}-m_{B}+g_{\sigma B} \sigma-g_{\omega B} \gamma_{\mu} \omega^{\mu}\right. \\
& \left.-\frac{1}{2} g_{\rho B} \gamma_{\mu} \tau \cdot \rho^{\mu}\right) \Psi_{B}+\frac{1}{2}\left(\partial_{\mu} \sigma \partial^{\mu} \sigma-m_{\sigma}^{2} \sigma^{2}\right) \\
& -\frac{1}{4} \omega_{\mu \nu} \omega^{\mu \nu}+\frac{1}{2} m_{\omega}^{2} \omega_{\mu} \omega^{\mu}-\frac{1}{4} \rho_{\mu \nu} \cdot \rho^{\mu \nu} \\
& +\frac{1}{2} m_{\rho}^{2} \rho_{\mu} \cdot \rho^{\mu}-\frac{1}{3} g_{2} \sigma^{3}-\frac{1}{4} g_{3} \sigma^{4} \\
& +\sum_{\lambda=e, \mu} \bar{\Psi}_{\lambda}\left(i \gamma_{\mu} \partial^{\mu}-m_{\lambda}\right) \Psi_{\lambda} \\
& +\mathcal{L}^{Y Y}+\mathcal{L}^{\delta} .
\end{aligned}
$$

The last two terms represent the contribution of the mesons $f \mathrm{~s}, \phi \mathrm{s}$ and $\delta \mathrm{s}$ and read

$$
\mathcal{L}^{Y Y}=\sum_{B} g_{f_{0} B} \bar{\Psi}_{B} \Psi_{B} f_{0}-\sum_{B} g_{\phi B} \bar{\Psi}_{B} \gamma_{\mu} \Psi_{B} \phi^{\mu}
$$




$$
\begin{aligned}
& +\frac{1}{2}\left(\partial_{\mu} f_{0} \partial^{\mu} f_{0}-m_{f_{0}}^{2} f_{0}^{2}\right) \\
& -\frac{1}{4} S_{\mu \nu} S^{\mu \nu}+\frac{1}{2} m_{\phi}^{2} \phi_{\mu} \phi^{\mu} .
\end{aligned}
$$

and

$$
\mathcal{L}^{\delta}=\sum_{B} \bar{\Psi}_{B} g_{\delta B} \tau \cdot \delta \Psi_{B}+\frac{1}{2} \partial_{\mu} \delta \partial^{\mu} \delta-\frac{1}{2} m_{\delta}^{2} \delta^{2}
$$

Here, $S_{\mu \nu}=\partial_{\mu} \phi_{\nu}-\partial_{\nu} \phi_{\mu}$.

For the condition of $\beta$ equilibrium, the chemical equilibrium is

$$
\mu_{i}=b_{i} \mu_{n}-q_{i} \mu_{e}
$$

where $b_{i}$ is the baryon number of a species $i$.

After the RMF approach is used, the energy density $\varepsilon$ and pressure $p$ of a PNS are given by 27, 28

$$
\begin{aligned}
\varepsilon= & \frac{1}{3} g_{2} \sigma^{3}+\frac{1}{4} g_{3} \sigma^{4}+\frac{1}{2} m_{\sigma}^{2} \sigma^{2}+\frac{1}{2} m_{\omega}^{2} \omega_{0}^{2}+\frac{1}{2} m_{\rho}^{2} \rho_{03}^{2} \\
& +\sum_{B} \frac{2 J_{B}+1}{2 \pi^{2}} \int_{0}^{\infty} \kappa^{2} \mathrm{~d} \kappa \sqrt{\kappa^{2}+m^{* 2}} \\
& \times\left(\exp \left[\left(\varepsilon_{B}(k)-\mu_{B}\right) / T\right]+1\right)^{-1} \\
& +\sum_{\lambda=e, \mu} \frac{2 J_{\lambda}+1}{2 \pi^{2}} \int_{0}^{\infty} \kappa^{2} \mathrm{~d} \kappa \sqrt{\kappa^{2}+m_{\lambda}^{2}} \\
& \times\left(\exp \left[\left(\varepsilon_{\lambda}(k)-\mu_{\lambda}\right) / T\right]+1\right)^{-1} \\
& +\frac{1}{2} m_{f}^{2} f_{0}^{2}+\frac{1}{2} m_{\phi}^{2} \phi_{0}^{2}+\frac{1}{2} m_{\delta}^{2} \delta_{0}^{2}, \\
p= & -\frac{1}{2} m_{\sigma}^{2} \sigma^{2}-\frac{1}{3} g_{2} \sigma^{3}-\frac{1}{4} g_{3}+\frac{1}{2} m_{\rho}^{2} \rho_{03}^{2} \sigma^{4}+\frac{1}{2} m_{\omega}^{2} \omega_{0}^{2} \\
& +\frac{1}{3} \sum_{B} \frac{2 J_{B}+1}{2 \pi^{2}} \int_{0}^{\infty} \frac{\kappa^{4}}{\sqrt{\kappa^{2}+m^{* 2}}} \mathrm{~d} \kappa \\
& \times\left(\exp \left[\left(\varepsilon_{B}(k)-\mu_{B}\right) / T\right]+1\right)^{-1} \\
& +\frac{1}{3} \sum_{\lambda=e, \mu} \frac{2 J_{\lambda}+1}{2 \pi^{2}} \int_{0}^{\infty} \frac{\kappa^{4}}{\sqrt{\kappa^{2}+m_{\lambda}^{2}}} \mathrm{~d} \kappa \\
& \times\left(\exp \left[\left(\varepsilon_{\lambda}(k)-\mu_{\lambda}\right) / T\right]+1\right)^{-1} \\
& -\frac{1}{2} m_{f}^{2} f_{0}^{2}+\frac{1}{2} m_{\phi}^{2} \phi_{0}^{2}-\frac{1}{2} m_{\delta}^{2} \delta_{0}^{2} . \\
& <
\end{aligned}
$$

where, $m^{*}$ is the effective mass of baryons

$$
m^{*}=m_{B}-g_{\sigma B} \sigma .
$$

The mass and the radius of a PNS can be calculated through the TolmanOppenheimer-Volkoff (TOV) equations 24, 25, 26. 


$$
\begin{aligned}
\frac{\mathrm{d} p}{\mathrm{~d} r} & =-\frac{(p+\varepsilon)\left(M+4 \pi r^{3} p\right)}{r(r-2 M)}, \\
M & =4 \pi \int_{0}^{R} \varepsilon r^{2} \mathrm{~d} r .
\end{aligned}
$$

The moment of inertia of a slowly rotating PNS is given by 24

$$
I=\frac{8 \pi}{3} \int_{0}^{R} \mathrm{~d} r r^{4} \frac{\varepsilon+p}{\sqrt{1-2 M(r) / r}} \frac{[\Omega-\omega(r)]}{\Omega} e^{-\nu} .
$$

Here, $\nu$ is given by

$$
-\frac{\mathrm{d} \nu(r)}{\mathrm{d} r}=\frac{1}{\varepsilon+p} \frac{\mathrm{d} p}{\mathrm{~d} r},
$$

and the angular velocity is given by

$$
-\frac{1}{r^{4}} \frac{\mathrm{d}}{\mathrm{d} r}\left(r^{4} j \frac{\mathrm{d} \bar{\omega}}{\mathrm{d} r}\right)+\frac{4}{r} \frac{\mathrm{d} j}{\mathrm{~d} r} \bar{\omega}=0 .
$$

The $j(r)$ is

$$
j(r)=e^{-(\nu+\lambda)}=e^{-\nu} \sqrt{1-2 M(r) / r}, r<R .
$$

The boundary condition are given by

$$
\begin{array}{r}
\frac{\mathrm{d} \bar{\omega}}{\mathrm{d} r}_{\mid r=0}=0, \\
\nu(\infty)=0 \\
\bar{\omega}(R)=\Omega-\frac{R}{3} \frac{\mathrm{d} \bar{\omega}}{\mathrm{d} r}{ }_{\mid r=R}
\end{array}
$$

\section{The parameters}

Because larger compression modulus $K$ will give the larger maximum mass of the NS [29, the nucleon coupling constant GM1, which compression modulus $K$ is larger, is chosen in this work [30]: the saturation density $\rho_{0}=0.153 \mathrm{fm}^{-3}$, binding energy $\mathrm{B} / \mathrm{A}=16.3 \mathrm{MeV}$, a compression modulus $K=300 \mathrm{MeV}$, charge symmetry coefficient $a_{\text {sym }}=32.5 \mathrm{MeV}$ and the effective mass $m^{*} / m=0.7$.

We define the ratios of hyperon coupling constant to nucleon coupling constant: $x_{\sigma h}=\frac{g_{\sigma h}}{g_{\sigma}}=x_{\sigma}, x_{\omega h}=\frac{g_{\omega h}}{g_{\omega}}=x_{\omega}, x_{\rho h}=\frac{g_{\rho h}}{g_{\rho}}$, with $h$ denoting hyperons $\Lambda, \Sigma$ and $\Xi$.

The calculations show that the ratio of hyperon coupling constant to nucleon coupling constant is in the range $1 / 3 \sim 1$ 30]. The maximum mass of the NS calculated will increase with the increase of the parameter $x_{\omega h}$ and so we choose $x_{\omega h}=0.9, .08,0.7, \ldots$, at first. The corresponding $x_{\sigma h}$ can be obtained by the hyperon well depth [2] 


$$
U_{h}^{(N)}=m_{n}\left(\frac{m_{n}^{*}}{m_{n}}-1\right) x_{\sigma h}+\left(\frac{g_{\omega}}{m_{\omega}}\right)^{2} \rho_{0} x_{\omega h} .
$$

Here, we choose $U_{\Lambda}^{(N)}=-30 \mathrm{MeV}$ 31, $U_{\Sigma}^{(N)}=+20 \mathrm{MeV}$ 32 and $U_{\Xi}^{(N)}=$ $-14 \mathrm{MeV}$ [33]. For the $x_{\rho h}$, we select $x_{\rho \Lambda}=0, x_{\rho \Sigma}=2, x_{\rho \Xi}=1$ by $\mathrm{SU}(6)$ symmetry 34 .

For the coupling constants of the mesons $f$ and $\phi$, we choose as 19

$$
\begin{gathered}
g_{f \Lambda} / g_{\sigma}=g_{f \Sigma} / g_{\sigma}=0.69, g_{f \Xi} / g_{\sigma}=1.25 \\
g_{\phi \Xi}=2 g_{\phi \Lambda}=2 g_{\phi \Sigma}=-2 \sqrt{2} g_{\omega} / 3 .
\end{gathered}
$$

The coupling constants of mesons $\delta$ are chosen as [20]

$$
g_{\delta \Sigma}=2 g_{\delta \Xi}=2 g_{\delta}, g_{\delta \Lambda}=0 .
$$

Here, the temperature is chosen as $T=20 \mathrm{MeV} 21$.

For the mesons $f, \phi$ and $\delta$ will soften the equation of state (EoS) and the softer EoS will give smaller PNS mass. Therefore, we calculate the mass of the PNS containing mesons $f, \phi$ and $\delta$ at first.

For parameters $x_{\omega h}=0.9,0.8$, we get two parameter sets: No.1 $\left(x_{\sigma \Lambda}=0.7961\right.$, $\left.x_{\omega \Lambda}=0.9 ; x_{\sigma \Sigma}=0.6186, x_{\omega \Sigma}=0.9 ; x_{\sigma \Xi}=0.7393, x_{\omega \Xi}=0.9\right)$ and No.2 $\left(x_{\sigma \Lambda}=0.7194\right.$, $\left.x_{\omega \Lambda}=0.8 ; x_{\sigma \Sigma}=0.5420, x_{\omega \Sigma}=0.8 ; x_{\sigma \Xi}=0.6627, x_{\omega \Xi}=0.8\right)$, by which the maximum mass obtained are $M_{\max }=2.249,2.139 \mathrm{M} \odot$, respectively (see Fig. 1). We hope to calculate the maximum mass of PNS as close as possible to the mass of the PNS PSR J0348+0432, where the influence of the mesons will be more obvious. But parameters No.01 and No.02 give the too larger maximum mass. So, we continue to select No.3 $\left(x_{\sigma \Lambda}=0.6428, x_{\omega \Lambda}=0.7 ; x_{\sigma \Sigma}=0.4653, x_{\omega \Sigma}=0.7 ; x_{\sigma \Xi}=0.5860\right.$, $x_{\omega \Xi}=0.7$ ), by which the maximum mass of the PNS calculated only is $2.005 \mathrm{M}_{\odot}$, still smaller than the mass of the PNS PSR J0348+0432. Then, we further choose No.4 $\left(x_{\sigma \Lambda}=0.6812, x_{\omega \Lambda}=0.75 ; x_{\sigma \Sigma}=0.5037, x_{\omega \Sigma}=0.75 ; x_{\sigma \Xi}=0.6244, x_{\omega} \Xi=0.75\right)$ and in this case we obtain the maximum mass $M_{\max }=2.074 \mathrm{M} \odot$, which is close to the mass of the PNS PSR J0348+0432.

Next, we use parameter No.04 to study the effect of $f_{\mathrm{s}}, \phi \mathrm{s}$ and $\delta \mathrm{s}$ on the moment of inertia of the PNS PSR J0348+0432.

\section{Effect of mesons $f, \phi$ and $\delta$ on energy density and pressure of the PNS PSR J0348+0432}

The contribution of mesons $f, \phi$ and $\delta$ to energy density $\varepsilon_{\delta}, \varepsilon_{f \phi}$ and $\varepsilon_{f \phi \delta}$ as a function of the baryon density $\rho$ are shown in Fig. 2 and Table 1. Here, $x_{\omega h}=0.75$.

From Table 11 we see that the central particle number density of the PNS PSR J0348 +0432 is in the range $\rho_{c}=0.526 \sim 0.618 \mathrm{fm}^{-3}$ as mesons $f, \phi$ and $\delta$ not being considered. When the mesons $\delta$ are included, the central particle number density decreases to $\rho_{c}=0.526 \sim 0.613 \mathrm{fm}^{-3}$ whereas it will increase to $\rho_{c}=0.542 \sim 0.665$ $\mathrm{fm}^{-3}$ as the mesons $f$ and $\phi$ being considered. Thereafter, the central particle number density will increase to $\rho_{c}=0.544 \sim 0.685 \mathrm{fm}^{-3}$ when the mesons $f, \phi$ and $\delta$ are all considered. 


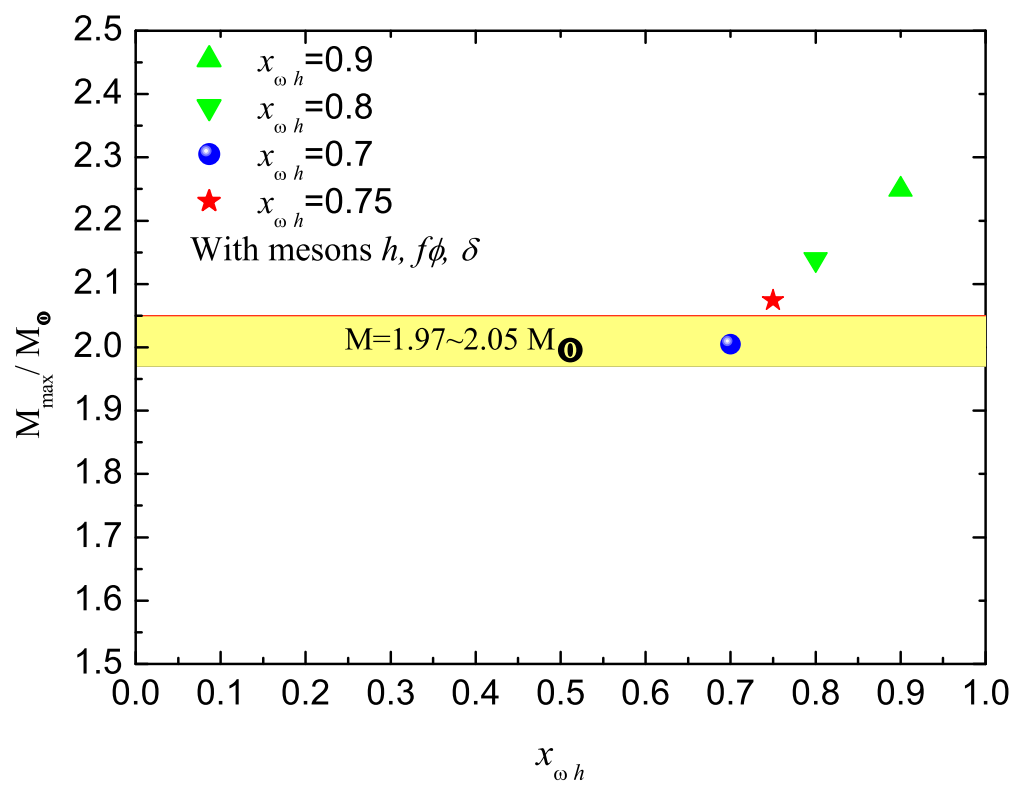

Fig. 1 The maximum mass $M_{\max }$ of the PNS as a function of the parameter $x_{\omega h}$.

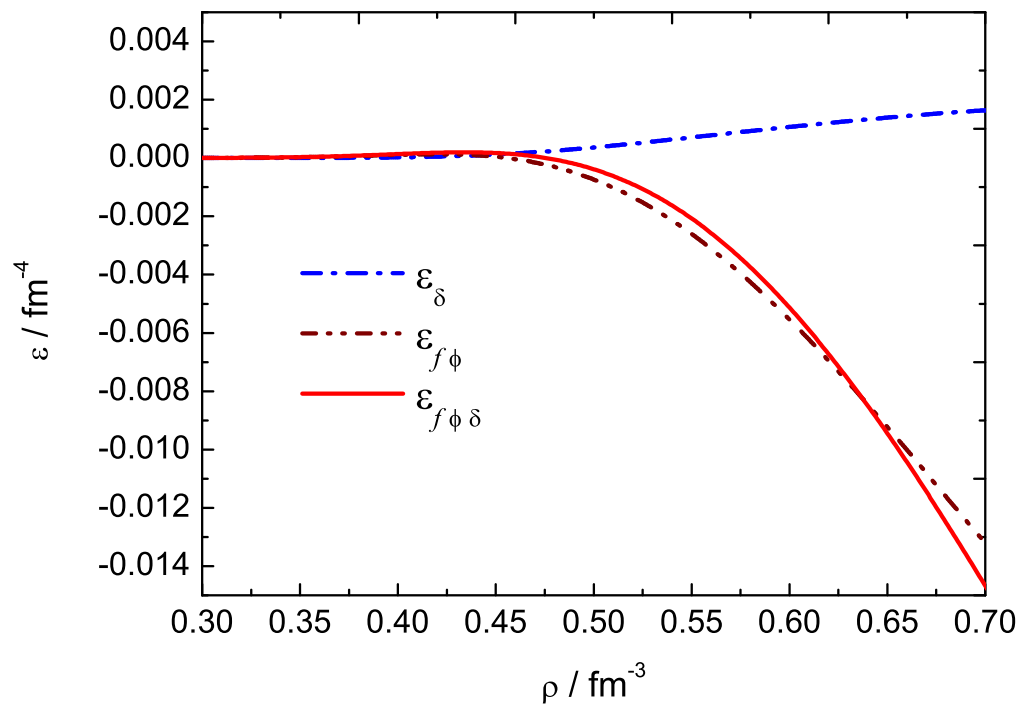

Fig. 2 The contribution of mesons $f, \phi$ and $\delta$ to energy density $\varepsilon_{\delta}, \varepsilon_{f \phi}$ and $\varepsilon_{f \phi \delta}$ as a function of the baryon density $\rho$. Here, $x_{\omega h}=0.75$. 
Table 1 The central particle number density $\rho_{c}$, the central energy density $\varepsilon_{c}, \varepsilon_{c, \delta}, \varepsilon_{c, f \phi}$, $\varepsilon_{c, f \phi \delta}$ and the central pressure $p_{c}, p_{c, \delta}, p_{c, f \phi}$ and $p_{c, f \phi \delta}$ of the PNS PSR J0348+0432 $M$. Here, $x_{\omega h}=0.75$. The $\varepsilon_{c}, \varepsilon_{c, \delta}, \varepsilon_{c, f \phi}, \varepsilon_{c, f \phi \delta}, p_{c}, p_{c, \delta}, p_{c, f \phi}$ and $p_{c, f \phi \delta}$ are in the units of fm ${ }^{-4}$, $\rho_{c} \mathrm{fm}^{-3}$,

\begin{tabular}{ccccc}
\hline Quantity & $h$ & $h \delta$ & $h f \phi$ & $h f \phi \delta$ \\
\hline$\rho_{c}$ & $0.526 \sim 0.618$ & $0.526 \sim 0.613$ & $0.542 \sim 0.665$ & $0.544 \sim 0.685$ \\
$\varepsilon_{c}$ & $2.857 \sim 3.473$ & $2.858 \sim 3.446$ & $2.960 \sim 3.789$ & $2.974 \sim 3.928$ \\
$p_{c}$ & $0.575 \sim 0.780$ & $0.575 \sim 0.775$ & $0.590 \sim 0.865$ & $0.590 \sim 0.900$ \\
\hline \hline Quantity & $h$ & $\delta$ & $f \phi$ & $f \phi \delta$ \\
\hline$\varepsilon_{c, \delta}$ & & $5.339 \mathrm{E}-4 \sim 1.15 \mathrm{E}-3$ & & \\
$\varepsilon_{c, f \phi}$ & & $-2.23 \mathrm{E}-3 \sim-1.041 \mathrm{E}-2$ & $-1.8 \mathrm{E}-3 \sim-1.304 \mathrm{E}-2$ \\
$\varepsilon_{c, f \phi \delta}$ & & & & \\
$p_{c, \delta}$ & & & & $-2.279 \mathrm{E}-2 \sim-4.968 \mathrm{E}-2$ \\
$p_{c, f \phi}$ & & & $-1.831 \mathrm{E}-2 \sim-3.225 \mathrm{E}-2$ & \\
$p_{c, f \phi \delta}$ & & & & \\
\hline
\end{tabular}

We see that the contribution of mesons $\delta$ to energy density $\varepsilon_{\delta}$ is greater than zero while the contribution of mesons $f$ and $\phi$ to energy density $\varepsilon_{f \phi}$ is less than zero. These mean that the energy density $\varepsilon$ will increase considering the mesons $\delta$ whereas it will decrease as the mesons $f$ and $\phi$ being considered. The magnitude of the decline is greater than the magnitude of the increase. Therefore, the contribution of the mesons $f, \phi$ and $\delta$ to the energy density $\varepsilon_{f \phi \delta}$ will be less than zero (see Fig. 2).

From Table 11 we also see the ratio of various central energy density is $\left|\varepsilon_{c}\right|$ : $\left|\varepsilon_{c, f \phi \delta}\right|:\left|\varepsilon_{c, f \phi}\right|:\left|\varepsilon_{c, \delta}\right| \cong 5000 \sim 6500: 3 \sim 24: 4 \sim 20: 1 \sim 2$. We see that the contribution of mesons $f$ and $\phi$ to central energy density is $4 \sim 10$ times larger than that of mesons $\delta$. But the contribution of mesons $f, \phi$ and $\delta$ to the energy density is still very small, only the energy density's $0.06 \sim 0.6 \%$ (see Fig. 3).

The central pressure $p_{c, \delta}, p_{c, f \phi}$ and $p_{c, f \phi \delta}$ of the PNS PSR J0348+0432 as a function of the baryon density $\rho$ are shown in Fig (also see Table. 11).

We see that the contribution of the mesons $\delta$ to pressure $p_{\delta}$ is greater than zero while the contribution of the mesons $f$ and $\phi$ to pressure $p_{f \phi}$ is less than zero. These mean that the pressure will increase considering the mesons $\delta$ and will decrease as the mesons $f$ and $\phi$ being considered. The magnitude of the decline is greater than that of the increase. Therefore, the contribution of the mesons $f, \phi$ and $\delta$ to the pressure $p_{f \phi \delta}$ will be less than zero (see Fig. 使).

We also see from Table 1 that the ratio of various central pressure is $\left|p_{c}\right|:\left|p_{c, f \phi \delta}\right|$ $:\left|p_{c, f \phi}\right|:\left|p_{c, \delta}\right| \cong 500 \sim 700: 20 \sim 50: 20 \sim 30: 1 \sim 7$. We see that the contribution of mesons $f$ and $\phi$ to central pressure is $20 \sim 4$ times larger than that of mesons $\delta$. But the contribution of mesons $f, \phi$ and $\delta$ to the central pressure is still very small, only the central pressure's $4 \sim 7 \%$ (see Fig. B). 

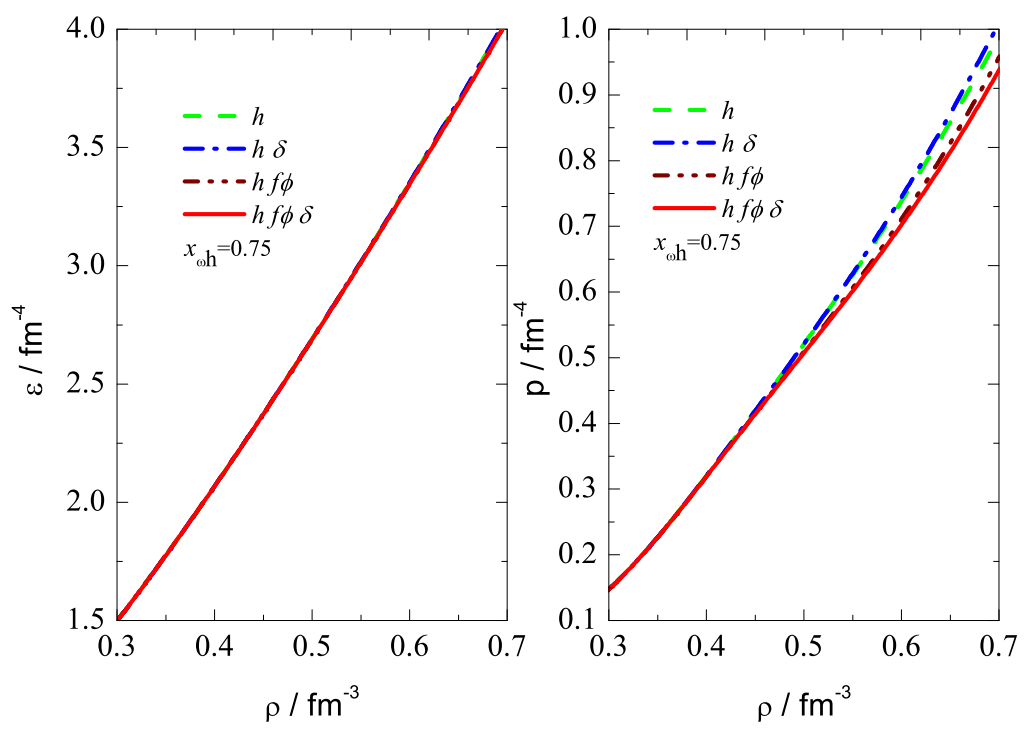

Fig. 3 The energy density $\varepsilon$ and pressure $p$ as a function of the baryon density $\rho$.

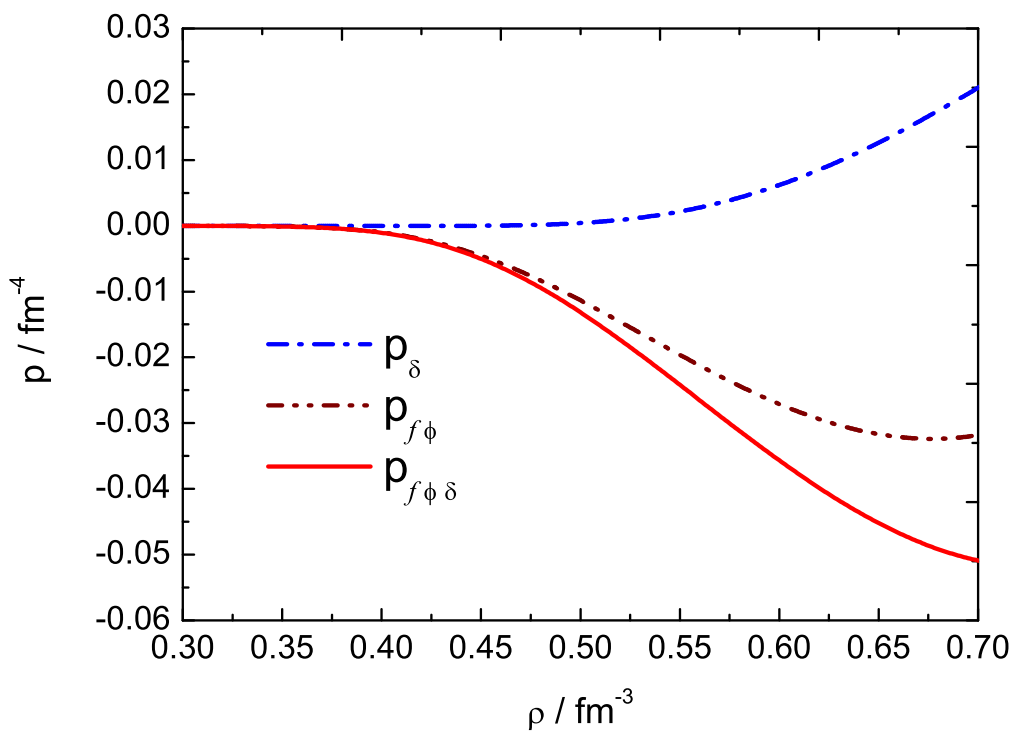

Fig. 4 The central pressure $p_{c, \delta}, p_{c, f \phi}$ and $p_{c, f \phi \delta}$ of the PNS PSR J0348+0432 as a function of the baryon density $\rho$. 
5 Effect of the mesons $f, \phi$ and $\delta$ on the radius $R$ of the PNS PSR J0348+0432

Figure 5 gives the radius $R$ as a function of the mass $M$ (also see Table 2). We see the radius increase as the mesons $\delta$ being considered whereas decrease when the mesons $f$ and $\phi$ included. Because the magnitude of the decline is larger than that of increase, the radius will decrease considered the contribution of mesons $f$, $\phi$ and $\delta$.

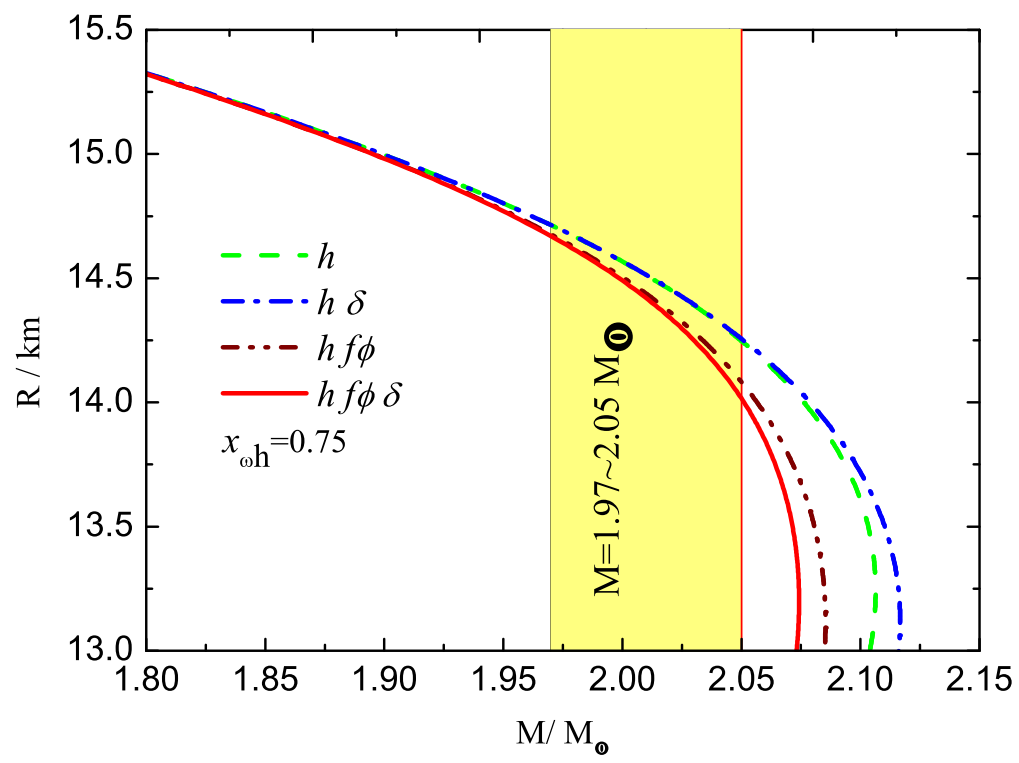

Fig. 5 The radius $R$ as a function of the mass $M$.

Table 2 The radius $R$, the moment of inertia of the PNS PSR J0348+0432 $I$ and the contribution of mesons $f, \phi$ and $\delta$ to the moment of inertia $I_{\delta}, I_{f \phi}$ and $I_{f \phi \delta}$. Here, $x_{\omega h}=0.75$. The $R$ is in the unit $\mathrm{km}, I, I_{\delta}, I_{f \phi}$ and $I_{f \phi \delta} \times 10^{45} \mathrm{~g} \mathrm{~cm}^{2}$.

\begin{tabular}{ccccc}
\hline Quantity & $h$ & $h \delta$ & $h f \phi$ & $h \delta f \phi$ \\
\hline$R$ & $14.708 \sim 14.246$ & $14.708 \sim 14.255$ & $14.670 \sim 14.080$ & $14.671 \sim 14.108$ \\
$I$ & $2.286 \sim 2.149$ & $2.287 \sim 2.161$ & $2.241 \sim 2.044$ & $2.233 \sim 2.003$ \\
\hline Quantity & $h$ & $\delta$ & $f \phi$ & $\delta f \phi$ \\
\hline$I_{\delta}$ & & $1.224 \mathrm{E} 42 \sim 1.161 \mathrm{E} 43$ & & \\
$I_{f \phi}$ & & & $-4.541 \mathrm{E} 43 \sim-1.057 \mathrm{E} 44$ & $-5.302 \mathrm{E} 43 \sim-1.465 \mathrm{E} 44$ \\
$I_{f \phi \delta}$ & & & & \\
\hline
\end{tabular}


Corresponding to the PNS PSR J0348+0432 $\left(M=1.97 \sim 2.05 \mathrm{M}_{\odot}\right)$, the radius is in the range $R=14.708 \sim 14.246 \mathrm{~km}$ as mesons $f, \phi$ and $\delta$ not being considered. When we consider mesons $\delta$ the radius increases to $R=14.708 \sim 14.255 \mathrm{~km}$ whereas decreases to $R=14.670 \sim 14.080 \mathrm{~km}$ as mesons $f$ and $\phi$ being considered. Thus, as mesons $f, \phi$ and $\delta$ all being considered the radius will decrease to $R=$ $14.671 \sim 14.108 \mathrm{~km}$.

\section{Effect of the mesons $f, \phi$ and $\delta$ on the moment of inertia of the PNS PSR J0348+0432}

The contribution of mesons $f, \phi$ and $\delta$ to the moment of inertia $I_{\delta}, I_{f \phi}$ and $I_{f \phi \delta}$ as a function of the mass $M$ are shown in Fig. 1 and Table 2 .

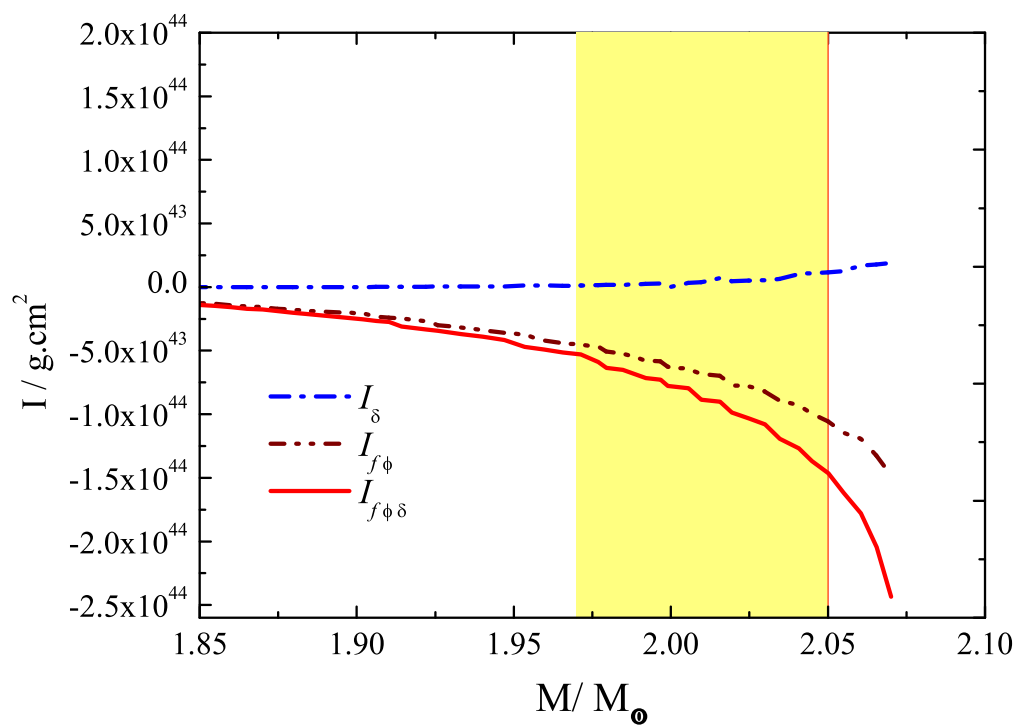

Fig. 6 The contribution of mesons $f, \phi$ and $\delta$ to the moment of inertia $I_{\delta}, I_{f \phi}$ and $I_{f \phi \delta}$ as a function of the mass $M$.

From Fig 6 we see that the contribution of mesons $\delta$ to moment of inertia $I_{\delta}$ is greater than zero whereas the contribution of mesons $f$ and $\phi$ to moment of inertia $I_{f \phi}$ is less than zero. That is to say that the moment of inertia $I$ will increase considering the mesons $\delta$ and will decrease as the mesons $f$ and $\phi$ being considered. For the magnitude of the decline is greater than the magnitude of the increase and therefore the contribution of mesons $f, \phi$ and $\delta$ to the moment of inertia $I_{f \phi \delta}$ will be less than zero (see Fig. 6).

From Table 2 we also see the ratio of various moment of inertia is $|I|:\left|I_{f \phi \delta}\right|$ : $\left|I_{f \phi}\right|:\left|I_{\delta}\right| \cong 1900 \sim 1800: 40 \sim 120: 40 \sim 90: 1 \sim 10$. We see that the contribution of 
mesons $f$ and $\phi$ to moment of inertia is $4 \sim 9$ times larger than that of mesons $\delta$. We also see that the contribution of mesons $f, \phi$ and $\delta$ to the moment of inertia is still very small, only the moment of inertia's $2 \sim 5 \%$ (see Fig. 7 ).

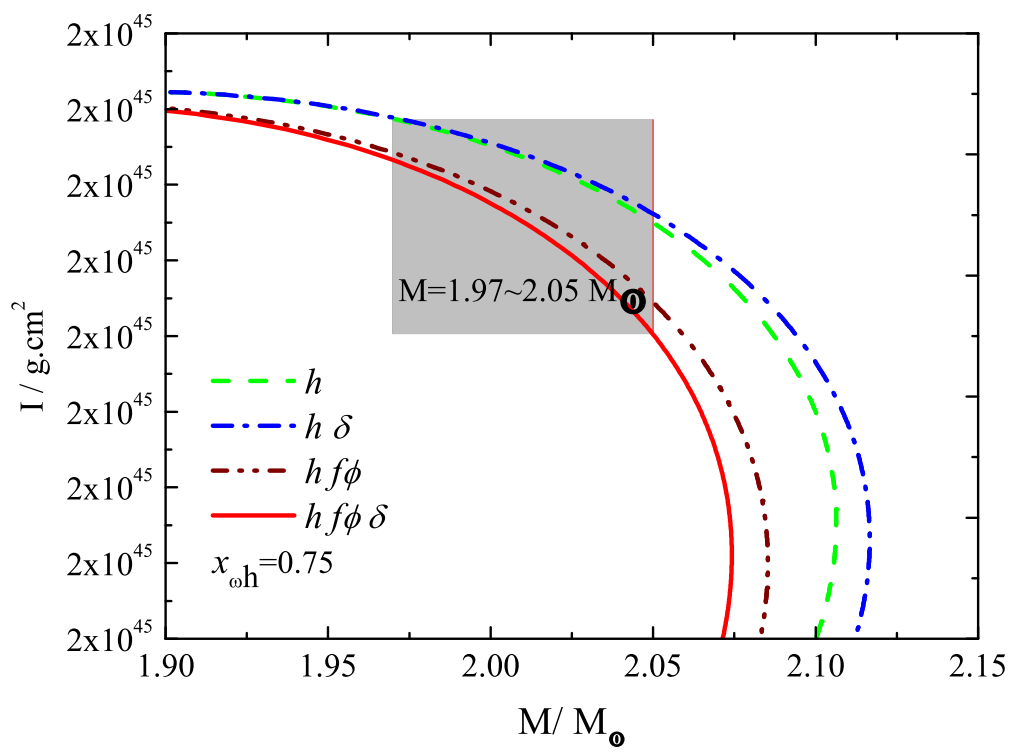

Fig. 7 The moment of inertia $I$ as a function of the mass $M$.

\section{Summary}

In this paper, we calculate the effect of $f_{0}(975) \mathrm{s}, \phi(1020) \mathrm{s}$ and $\delta \mathrm{s}$ on the moment of inertia of the PNS PSR J0348+0432 in the framework of the RMF theory considering the baryon octet. In this work, we choose the nucleon coupling constants GM1, the hyperon well depth $U_{\Lambda}^{(N)}=-30 \mathrm{MeV}, U_{\Sigma}^{(N)}=+20 \mathrm{MeV}, U_{\Xi}^{(N)}=-14 \mathrm{MeV}$ and the temperature $T=20 \mathrm{MeV}$.

We find that the contributions of mesons $\delta$ to energy density $\varepsilon_{\delta}$ and pressure $p_{\delta}$ are greater than zero while the contributions of mesons $f$ and $\phi$ to energy density $\varepsilon_{f \phi}$ and pressure $p_{f \phi}$ are less than zero. These cause the energy density $\varepsilon$ and pressure $p$ increase considering the mesons $\delta$ whereas will decrease as the mesons $f \phi$ being considered. Because of the magnitude of the decline being greater than the magnitude of the increase, the $\varepsilon_{f \phi \delta}$ will decrease considering the mesons $f, \phi$ and $\delta$.

The contribution of mesons $f$ and $\phi$ to central energy density is $4 \sim 10$ times larger than that of mesons $\delta$ and the contribution of mesons $f$ and $\phi$ to central 
pressure is $20 \sim 4$ times larger than that of mesons $\delta$. We also see that the contribution of mesons $f, \phi$ and $\delta$ to the central energy density is only the energy density's $0.06 \sim 0.6 \%$ but the contribution of mesons $f, \phi$ and $\delta$ to the central pressure is the central pressure's $4 \sim 7 \%$.

The radius increase as the mesons $\delta$ being considered whereas decrease as the mesons $f$ and $\phi$ being included. The magnitude of the decline being larger than that of the increase leads to the radius decrease when the contributions of mesons $f, \phi$ and $\delta$ are considered.

Similarly, the contribution of mesons $\delta$ to moment of inertia $I_{\delta}$ is greater than zero whereas the contribution of mesons $f$ and $\phi$ to moment of inertia $I_{f \phi}$ is less than zero, i.e. the moment of inertia $I$ will increase considering the mesons $\delta$ whereas will decrease as the mesons $f$ and $\phi$ being considered. As a result of the magnitude of the decline being greater than the magnitude of the increase the $I_{f \phi \delta}$ will decrease considering the mesons $f, \phi$ and $\delta$. In addition, we also find that the contribution of mesons $f$ and $\phi$ to moment of inertia is $4 \sim 9$ times larger than that of mesons $\delta$ and the mesons $f, \phi$ and $\delta$ contribute to the moment of inertia's $2 \sim 5 \%$.

Acknowledgements We are thankful to Shan-Gui Zhou for fruitful discussions during my visit to the Institute of Theoretical Physics of Chinese Academy of Sciences. This work was supported by the Natural Science Foundation of China (Grant No. 11447003 ) and the Scientific Research Foundation of the Higher Education Institutions of Anhui Province, China ( Grant No. KJ2014A182 ).

\section{References}

1. Hartle, J.B.: Astrophys. J. 150, 1005(1967).

2. Hartle, J.B., Thorne, K.S.: Astrophys. J. 153, 807(1968).

3. Bejger, M., Bulik, T., Haensel, P.: Monthly Notices of the Royal Astronomical Society 364, 635(2005)

4. Raithel, C.A., Özel, F., Psaltis, D.: Phys. Rev. C 93, 032801(2016).

5. Demorest, P.B., Pennucci, T., Ransom, S.M., et al.: Nature 467, 1081(2010).

6. Antoniadis, J., Freire, P.C.C., Wex, N., Tauris, T.M., Lynch, R.S., van Kerkwijk, M.H., Kramer, M., Bassa, C., Dhillon, V. S., Driebe, T., Hessels, J.W.T., Kaspi, V.M., Kondratiev, V.I., Langer, N., Marsh, T.R., McLaughlin, M. A., Pennucci, T.T., Ransom, S.M., Stairs, I.H., van Leeuwen, J., Verbiest, J.P.W., Whelan, D.G.: Science, 340, 448(2013).

7. Massot, É., Margueron, J., Chanfray, G.: Europhysics Letters 97(3), 39002(2012).

8. Masuda, K., Hatsuda, T., Takatsuka, T.: Astrophys. J. 764,12(2012).

9. Mallick, R.: Phys. Rev. C 87, 025804(2013)

10. Whittenbury, D.L., Carroll, J.D., Thomas, A.W., Tsushima, K., Stone, J.R.: eprint arXiv: 1204.2614(2012).

11. Jiang, W.Z., Li, B.A., Chen, L.W.: Astrophys. J. 756, 56(2012).

12. Weissenborn, S., Chatterjee, D., Schaffner-Bielich, J.: Phys. Rev. C 85(6), 065802(2012).

13. Chamel, N., Fantina, A.F., Pearson, J.M., Goriely, S.: Astronomy \& Astrophysics 553, A22(2013).

14. Katayama, T., Miyatsu, T., Saito, K.: The Astrophysical Journal Supplement 203, $22(2012)$.

15. Weissenborn, S., Chatterjee, D., Schaffner-Bielich, J.: Nucl. Phys. A 881, 62(2012).

16. Bednarek, I., Haensel, P., Zdunik, J. L., Bejger, M., Mańka, R.: Astronomy \& Astrophysics 543, A157(2012).

17. Zhou, S. G.: Phys. Scr., 91, 063008(2016)

18. Glendenning, N. K.: Astrophys. J. 293, 470(1985).

19. Schaffner, J., Dover, C. B., Gal, A.: Ann. Phys. 235, 35(1994).

20. Menezes, D. P., Providência, C.: Phys. Rev. C, 70, 058801(2004). 
21. Burrows, A., Lattier, J. M.: Astrophy. 307, 178(1986).

22. Hofmann, F., Keil, C. M., Lenske, H.: Phys. Rev. C 64, 034314(2001).

23. Kubis, S., Kutschera, M.: Phys. Lett. B 399, 191(1997).

24. Glendenning, N. K.: Compact Stars: Nuclear Physics, Particle Physics, and General Relativity, Springer-Verlag, New York, Inc, 1997.

25. Tolman, R.C.: Phys. Rev., 55, 364(1939).

26. Oppenheimer, J. R., Volkoff, G. M.: Phys. Rev., 55, 374(1939).

27. Glendenning, N. K.: Phys. Lett. B, 185, 275(1987).

28. Glendenning, N. K.: Nucl. Phys. A, 469, 600(1987).

29. Zhao, X. F., Dong, A. J., Jia, H. Y.: Acta Physica Polonica B, 43, 1783(2012).

30. Glendenning, N. K., Moszkowski, S. A.: Phys.Rev.Lett. 67, 2414(1991).

31. Schaffner-Bielich, J., Gal, A.: Phys. Rev. C 62, 034311(2000).

32. Kohno, M., Fujiwara, Y., Suzuki, Y.: Nuclear Physics A, 835, 358(2010).

33. Harada, T., Hirabayashi, Y., Umeya, A.: Physics Letters B, 690, 363(2010).

34. Schaffner, J., Mishustin, I. N.: Phys. Rev. C 53, 1416(1996).

35. Bejger, M., Haensel, P.: Astronomy and Astrophysics, 396, 917(2002).

36. Lattimer, J. M., Schutz, B. F.: Astrophys. J., 629, 979(2005). 